\title{
Adaptation-induced collective dynamics of a single-cell protozoan
}

Article

Published Version

Ogata, M., Hondou, T., Hayakawa, Y., Hayashi, Y. and Sugawara, K. (2008) Adaptation-induced collective dynamics of a single-cell protozoan. Physical Review E, 77. 011917. ISSN 1550-2376 doi:

https://doi.org/10.1103/PhysRevE.77.011917 Available at https://centaur.reading.ac.uk/30816/

It is advisable to refer to the publisher's version if you intend to cite from the work. See Guidance on citing.

To link to this article DOI: http://dx.doi.org/10.1103/PhysRevE.77.011917

Publisher: American Physical Society

All outputs in CentAUR are protected by Intellectual Property Rights law, including copyright law. Copyright and IPR is retained by the creators or other copyright holders. Terms and conditions for use of this material are defined in the End User Agreement.

\section{www.reading.ac.uk/centaur}

\section{CentAUR}

Central Archive at the University of Reading 
Reading's research outputs online 


\title{
Adaptation-induced collective dynamics of a single-cell protozoan
}

\author{
Maiko Ogata, Tsuyoshi Hondou, and Yoshinori Hayakawa* \\ Department of Physics, Tohoku University, Sendai 980-8578, Japan
}

Yoshikatsu Hayashi and Ken Sugawara

Faculty of Liberal Arts, Tohoku Gakuin University, Sendai 981-3193, Japan

(Received 2 August 2007; revised manuscript received 18 October 2007; published 23 January 2008)

\begin{abstract}
We investigate the behavior of a single-cell protozoan in a narrow tubular ring. This environment forces them to swim under a one-dimensional periodic boundary condition. Above a critical density, single-cell protozoa aggregate spontaneously without external stimulation. The high-density zone of swimming cells exhibits a characteristic collective dynamics including translation and boundary fluctuation. We analyzed the velocity distribution and turn rate of swimming cells and found that the regulation of the turing rate leads to a stable aggregation and that acceleration of velocity triggers instability of aggregation. These two opposing effects may help to explain the spontaneous dynamics of collective behavior. We also propose a stochastic model for the mechanism underlying the collective behavior of swimming cells.
\end{abstract}

DOI: 10.1103/PhysRevE.77.011917

\section{INTRODUCTION}

Many microorganisms under water cluster in groups. In general, in order to generate cooperative collective behavior, they utilize several kinds of physical field which they can produce or perturb themselves. Chemotaxis is one of the most typical examples [1].

When the coupling between cells and these external fields is appropriate, the cells exhibit a variety of dynamical motions as a group, rather than simply cluster into fixed positions. For example, in the chemotaxis of Escherichia coli, Tsimring et al. proposed a reaction-diffusion model that could describe various type of spot patterns seen in colonies by introducing interaction of cells to their by-products [2]. Salman et al. recently found a translation mode of the aggregation in a temperature gradient [3], and they concluded that the solitary mode of aggregation was the result of a feedback mechanism provided by interbacterial communication utilizing a chemical field.

In a macroscopic description of such types of organisms with a large population, the internal cellular dynamics, which could be considered as a form of adaptation, is often neglected, because it is expected that such a dynamics is fast enough in the sense of "slave modes," which are assumed in reaction-diffusion models such as the Keller-Segel model for chemotaxis $[4,5]$.

In spite of success of these theories, the environment has influences on the behavior of cell via its internal state. When the rate change of the internal state is not fast enough to follow the change of environment, the internal state depends on the past as well as the present environment [6]. Therefore, when the characteristic time scale of internal degrees of freedom becomes comparable to that of cell motion, another dynamics arising from the collective behavior might emerge.

The paramecium is one of the largest single-cell protozoa commonly living in fresh water. The size of paramecium

\footnotetext{
*hida@cmpt.phys.tohoku.ac.jp
}

PACS number(s): 87.17.Jj, 05.40.Fb, 87.17.Aa, 87.18.Hf

multimicronucleatum is about $0.2 \mathrm{~mm}$ in its longitudinal axis. Its typical swimming velocity is $0.5 \mathrm{~mm} / \mathrm{s}$ [10], and the rate of directional change is a few times in a minute under normal conditions. The swimming behavior of paramecium depends on its ciliary motion; the stroke of cilia drives the cell forward with left-handed rotation around its longitudinal axis, which leads to a left-handed spiral path [9].

Paramecia are known to react to several types of external stimuli such as the presence of certain chemical substances, temperature change, or an electric field. For example, if cells sense a chemical attractant, they swim faster to get into the attractant. On the contrary, if they sense a chemical repellent, they stop or swim backward and make turns to start swimming again by controlling the beat of their cilia $[7,8]$.

For paramecia, this response to their environment can also be modified significantly by their internal state. If paramecia experience a sudden temperature drop, they react immediately by increasing their turn rates, however, the swimming velocity decreased gradually parallel to the magnitude of temperature. After a certain time, typically of the order of minutes, their turn rate return to normal, due to adaptation to the new temperature. When paramecia are placed in a temperature gradient, they accumulate at the optimal temperature, the growth temperature. This accumulation in the temperature gradient is the result of the differences in their turn rates ascending and descending the gradient. On the other hand, swimming velocity is proportional to the magnitude of temperature, independently of the swimming direction [12].

In this paper, we examine the behavior of paramecia in a simple quasi-one-dimensional geometry as an example of collective dynamics intermediated by both chemical signaling and the adaptation process of each individual cell. Paramecia exhibit not simply a stable aggregation forming process but also a dynamic swarming motion in our experiment. We also present a phenomenological model, based on our experimental results, which includes simple internal dynamics. 


\section{EXPERIMENTS}

We cultured a wild type of paramecium multimicronucleatum. Paramecia were cultivated in a straw-infusion medium as described in Ref. [11]. The cultures were incubated in a $25{ }^{\circ} \mathrm{C}$ water bath. Only robust cells in log-phase growth were used in the following experiments.

To obtain suspension at different cell densities, we applied a centrifuge technique for condensation and exchanged the cultured solution, in order to eliminate contaminating bacteria and dust. Next, we injected the prepared suspension into a narrow transparent tube (Tygon R-3603) having an inner diameter of $0.79 \mathrm{~mm}$ and a length of $60 \mathrm{~mm}$ by using a syringe. After injection, the ends of the flexible tube were connected with a joint tube to form a ring. This environment forced them to swim under a quasi-one-dimensional periodic boundary condition so that effects that appear in higher dimensions such as geotaxis and convection of water could be neglected. The sample was video recorded by CCD camera under uniform lighting and the temperature was regulated (at $26 \pm 0.1^{\circ} \mathrm{C}$ ) for several hours. During data acquisition, cell division occurred only rarely so that the cell population was conserved. By analyzing the movie we obtained trajectories of each cell, which yielded their density, velocity, and turn rate.

When the prepared cell density was larger than that of a critical concentration (approximately one cell per $\mathrm{mm}^{3}$ ), most of the swimming cells localized from a homogeneous distribution ended up as an aggregation. As a consequence, the density profile had a steep slope between low- and highdensity regions. We assume that this steep slope corresponds to the "invisible boundary" reported by Jennings in the study of chemotaxis of paramecia [8]. He found that paramecia in a water on a slide did not remain scattered and formed an aggregation, although quantitative conditions for the aggregation forming were not discussed. Within the aggregation the individual cells moved about randomly. However, on coming to an invisible outer boundary, they gave the avoiding reaction by making turns. In the case of present onedimensional geometry, we observed the high-density region as a "belt."

As the prepared density increased, behavior of the aggregation became more dynamic as illustrated in Fig. 1. When the average density increased to more than 3 cells $/ \mathrm{mm}^{3}$, the belt started to move translationally as seen in Fig. 2(a) at a very low velocity compared to the swimming velocity of individual cells. We refer to this motion as a "translational belt." The density profile had a steep slope between the low and high-density regions, and was uniform between the boundaries.

When the cell density was increased further, the belt exhibited a more irregular dynamics [Fig. 2(b)]. The size of the aggregation and the cell density within the belt were no longer constant and a high-density spot was formed in the belt. In this high-density area, the swimming cells occasionally made physical contact. We also observed both annihilation and nucleation of the belt over a long time scale. To reveal the mechanism of the collective dynamics of swimming cells, we analyzed the behavior of individual cells by focusing on the turn rate and velocity at different positions.

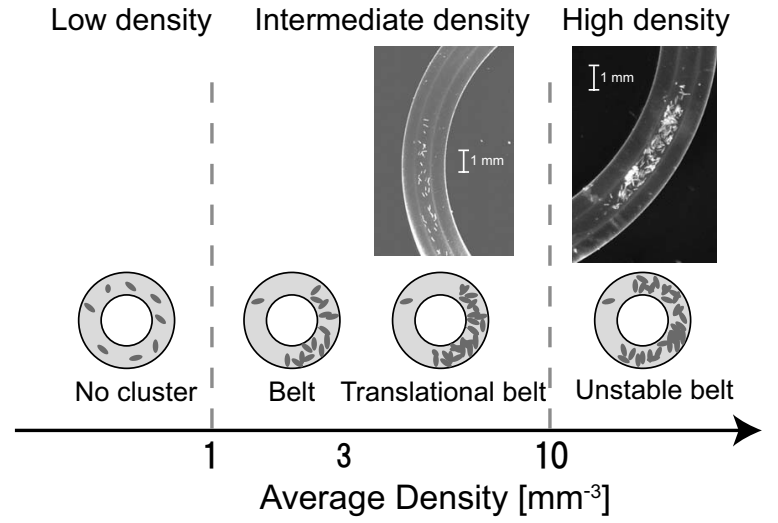

FIG. 1. Schematic diagram of the type of aggregation seen at different cell densities and snapshots of swimming cells in a ring made of silicon rubber.

We plotted the turn rates at small segments along the ring in Fig. 3, where $f_{+}$and $f_{-}\left[\mathrm{s}^{-1}\right]$ signify turn rates of cells swimming in the positive and negative directions, respectively. While very few cells turned in the region where the density was almost constant, at regions in which the density varied a great deal, $f_{+}$and $f_{-}$increased significantly. Outside the aggregation there was no apparent variation in turn rate. This implies that the probability of swimming out of the aggregation is selectively reduced, leading to the formation of aggregations.

Since it is known that paramecia are sensitive to chemical environment parameters such as $\mathrm{pH}$ and carbon dioxide concentration, it is plausible to assume that they change their

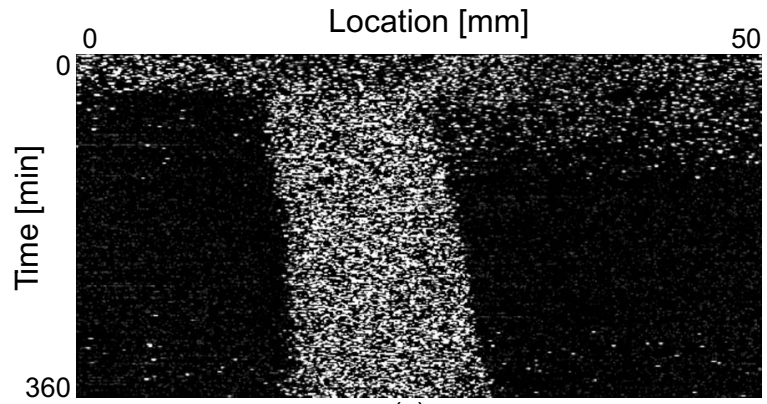

(a)

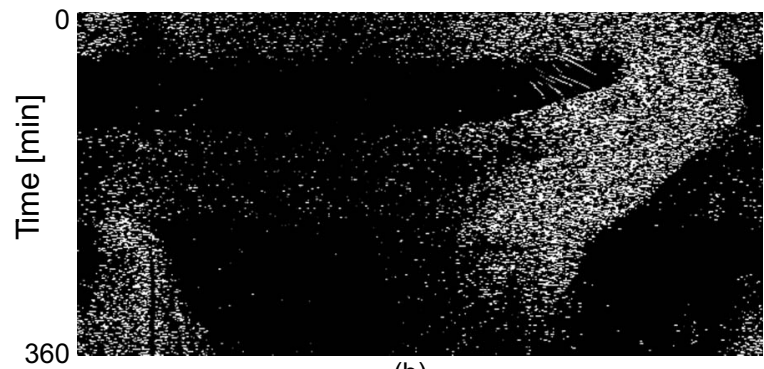

(b)

FIG. 2. Time evolution of cell density along the tube. The horizontal axis denotes the curvilinear location $(\mathrm{mm})$ along the tube. A snapshot of the aggregation was taken every $30 \mathrm{~s}$ for $360 \mathrm{~min}$. Each image was subtracted from the one following in order to detect only swimming objects. Average cell density in the tube was (a) 3.0 and (b) $12 \mathrm{~mm}^{-3}$. 


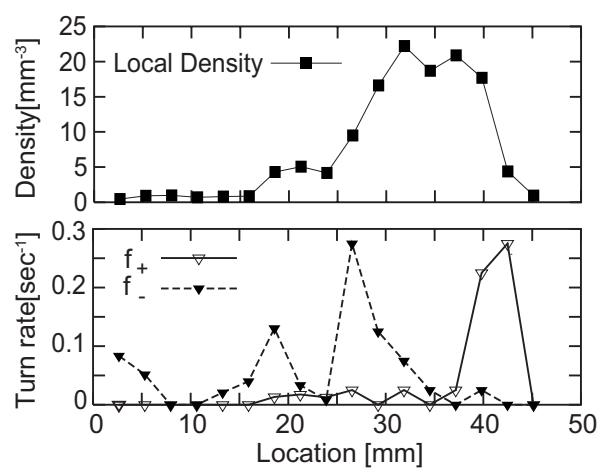

FIG. 3. Local density along the tube (top) and turn rates of cells swimming towards each positive and negative direction (bottom). Turn rates of swimming cells in the direction of decreasing density gradient increased selectively.

turn rate due to the chemical gradient of some metabolite they produce, however, such chemicals have not yet been identified directly. We note that paramecia are known to gather due to a certain concentration of externally added acetic acid [8]. In our experiments, we found that carbon dioxide gas injected into a culture medium also induced an aggregation even when the cell density of the medium was very low. We expect that carbon dioxide produced by cells can be the possible chemo-attractant which affects turn rate.

Paramecia control not only their turn rate but also vary their swimming velocity significantly. The velocity distributions outside and inside the aggregation are shown in Figs. 4(b) and 4(c). Inside the aggregation, the average velocity was $0.7 \mathrm{~mm} / \mathrm{s}$ and the distribution was narrow. On the other hand, outside the region, the average velocity was more than twice as great $(2.0 \mathrm{~mm} / \mathrm{s})$ with a larger variance. From the observation of swimming trajectories, some population of cells which reenter the aggregation with high velocity hold the velocity until they make a turn. In Fig. 4(c), such fast swimming cells are counted.

Tracking of cells near the boundary region indicated a selective acceleration and deceleration mechanism that resulted in the swimming velocity distribution depending on position. In our observation, when a cell was moving out from the aggregation, it increased its swimming velocity and kept this higher velocity in the lower density region [Fig. 5(a)]. Cells in the aggregation that reentered the aggregation by making a turn showed a sudden acceleration near the boundary region. However, after their reentrance into the aggregation, their swimming velocity returned to its stationary value. Observation also showed that cells entering from the low-density region with high velocity into the aggregation exhibited rapid deceleration near the boundary region [Fig. $5(b)$. Velocity change occurs mainly at the timing of turns, and some population of cells comming into the aggregation with high velocity persistently swim faster even in the dense region until they make a turn.

To elucidate the effect of inhomogeneous cell density on the velocity distribution, we injected a single cell into the tube and analyzed its behavior. As seen in Fig. 4(a), a swimming cell has a certain velocity fluctuation. By comparing the velocity distribution of single cell [Fig. 4(a)] with those
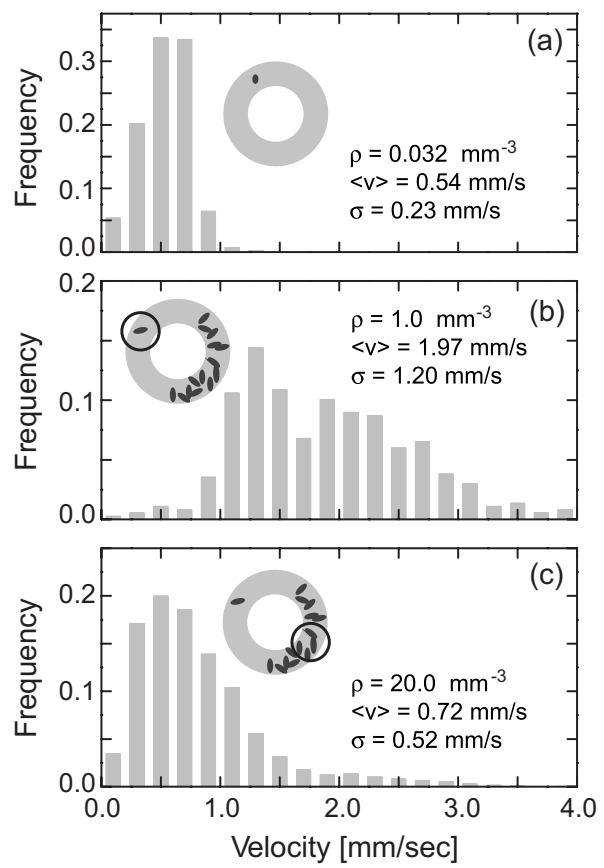

FIG. 4. Velocity distribution of swimming cells: (a) A single cell injected into the ring showed some velocity fluctuation. When the population of cells was large, distributions at low-density regions (b) and high-density regions (c) became significantly differentiated. Cells in the dense region swam similar to a single free cell. Average cell density $\rho$, swimming velocity $\langle v\rangle$, and standard deviation of the velocity $\sigma$ are inserted.

inside an aggregation [Fig. 4(c)], it is clear that the distribution for a single cell resembled that of cells inside the aggregation, even though the cell density in this case was closer to that outside the region of aggregation.

In Fig. 6, we plot the average swimming velocity at different positions having various local densities, in the case that the belt structure was observed. Dense parts correspond to the aggregation, where average swimming velocity was almost constant (about $0.7 \mathrm{~mm} / \mathrm{s}$ ) having the same magnitude as that for a single paramecium without external stimuli. This plot indicates that, even in the high-density region, volume interactions could be considered to have a negligible effect on swimming behavior. In fact, intercell distance in this region was still several times larger than their body size, so that the aggregation should not be considered as a traffic jam of bidirectional flow [13]. At low-density regions, outside the aggregation, the average velocity increased significantly.

\section{DISCUSSIONS AND MODELING}

We interpret the above experimental results in the following way: for an isolated cell, the environment along the tube is uniform no matter where or in which direction it swims. Therefore such a cell should be adapted to an environment with lower cell density. For cells in a tube where an aggregation exists, the chemical composition will be different inside and outside the aggregation. Due to the turn rate control 

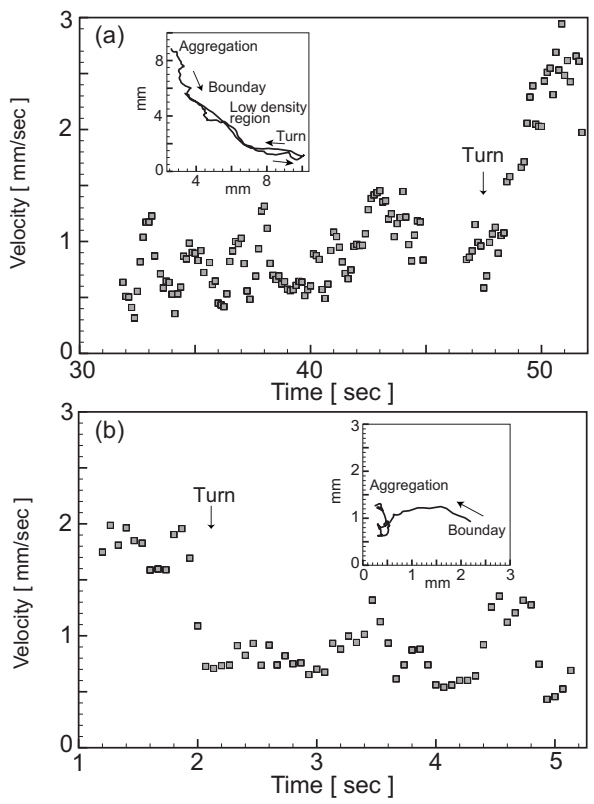

FIG. 5. Trace of swimming velocity and trajectoy (inset) of single cell near boundary region of the aggregation. (a) Escaping cell from the aggregation acclerate gradulally across the boundary and raise the velocity suddenly after making a turn in the lowdensity region. (b) Reentered cell from the low-density region decreases the velocity to the stationary level after a turn.

mechanism as seen in Fig. 3, swimming cells tend to be confined inside the aggregation. We can expect that those cells will adapt to the chemical environment in the aggregation because of longer sojourn time. If such a cell happens to swim out of the aggregation into a region with a different chemical environment to which it is not adapted, the sudden change in chemical composition causes stress and the cell swims twice as fast.

The formation of an aggregate can be regarded as a selforganized clustering process that may consist of two opposing effects; at first, the swimming cells respond to the chemical gradient instantaneously by regulating their turn rate. This leads to the formation of a stable aggregate within a quarter of an hour. Secondly, individual cells accelerate when they escape from the aggregate, presumably due to chemical

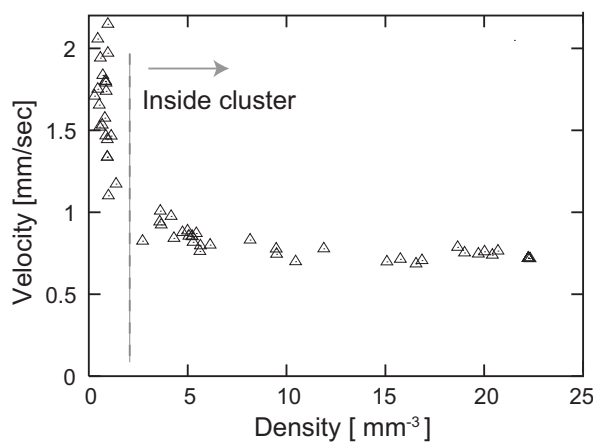

FIG. 6. Average swimming velocity versus cell density in different parts of the ring. Average velocity became constant at highdensity regions (inside the cluster) and increased significantly at low-density regions (outside the cluster). stress. The memory of the stress would be sustained, at least, for the time that a cell turns around the ring (typically $30 \mathrm{~s}$ ). The acceleration of the swimming cell may work to destabilize the cluster.

These two opposing effects of turn rate and velocity regulation may play a significant role for the formation of the mobile belt as shown in Fig. 2. To clarify this point, we propose a stochastic model for swimming cells in terms of random walkers interacting through chemical signals with a minimal internal memory effect. For simplicity we consider only the one-dimensional case. It is easy to generalize the present model to higher dimensional cases.

We assume that element $i$ representing a swimming cell moves at velocity $v_{i}$ emitting a chemoattractant at constant rate $\zeta$. The concentration $c$ of the chemical follows a diffusion equation with a decay term as follows:

$$
\frac{\partial c}{\partial t}=D_{\mathrm{a}} \nabla^{2} c-\alpha c+\zeta \sum_{i} \delta\left(x-x_{i}\right)
$$

where $x_{i}$ denotes the position of the $i$ th element.

Each element makes turns stochastically at a turn rate that depends on the gradient of the chemoattractant and the swimming direction $n_{i}=v_{i} /\left|v_{i}\right|$ as follows:

$$
\mu_{i}=\bar{\mu}\left[1+\lambda f\left(n_{i} \cdot \nabla c\right)\right],
$$

where we assume the following asymmetric sensitivity to the chemical gradient:

$$
f(\xi)= \begin{cases}\frac{|\xi|}{k_{a}+|\xi|}, & \xi<0, \\ 0, & \xi \geq 0 .\end{cases}
$$

These elements interact only through the chemical field, and neither excluded volume interactions nor fluid effects are taken into account.

If the swimming velocity $v_{i}$ remains constant $\left(v_{i}=\bar{v}\right)$, the collective dynamics of elements described by the above model will simply exhibit an aggregation process which can be fairly described utilizing the Keller-Segel model of chemotaxis. As shown in Fig. 8(a) and 8(c), several spots of greater population density are formed, resulting eventually in a single stable cluster.

To account for the velocity control mechanism, possibly due to chemical stress, seen in our experiments, we introduce a simple internal degree of freedom $\Phi_{i}$ for each swimming organism to represent an adaptation process to a chemical environment. We assume that the internal dynamics of $\Phi_{i}$ simply follows the chemical concentration of chemoattractant with a characteristic memory-loss time, i.e., an adaptation time $\tau$, thus

$$
\tau \frac{d \Phi_{i}}{d t}=-\Phi_{i}+c\left(x_{i}, t\right)
$$

Due to this relaxation process, the internal memory $\Phi_{i}$ will slowly approach to the surrounding chemical concentration $c$, if $\tau$ is large.

To estimate the adaptation time $\tau$ of the cells in the tube, we calculated the autocorrelation function of magnitude of 


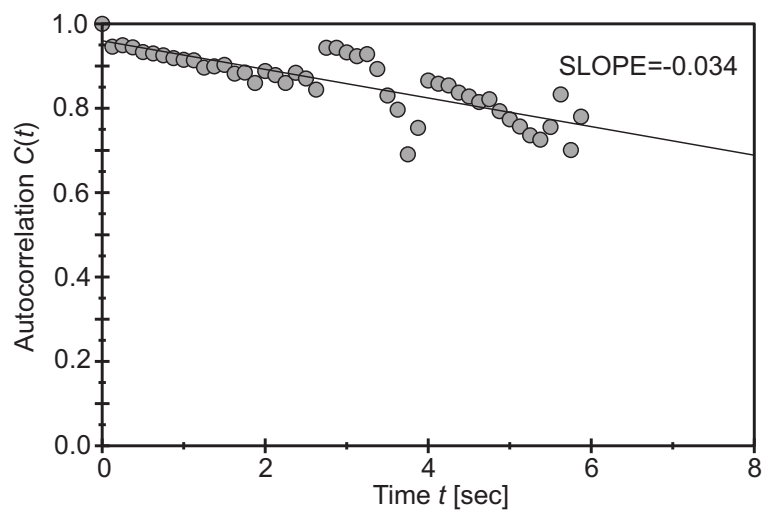

FIG. 7. Autocorrelation function of swimming velocity for paramecia in the low-density region. Trajectories of 34 paramecia between turns were analyzed. The curvilinear length along the ring subject to the analysis was $14 \mathrm{~mm}$, and average cell density was $5 \mathrm{~mm}^{-3}$. From the slope of the plot, decay time is estimated as $30 \mathrm{~s}$.

velocity $C(t)=\left\langle v\left(t^{\prime}+t\right) v\left(t^{\prime}\right)\right\rangle /\left\langle v\left(t^{\prime}\right)^{2}\right\rangle$ for the cells swimming in the low-density region at the opposite side of the belt in the ring (Fig. 7). Although we could keep track of individual cells only for $6 \mathrm{~s}$ long at most from the limitation of the field of view in microscope, the autocorrelation clearly shows a slow decay. If we assume an exponential decay, the memory time is expected to be about $30 \mathrm{~s}$ for this sample, which is significantly longer than turning intervals.

We assume that each element controls its swimming velocity according to the "chemical stress" which it feels instantaneously at its position as

$$
v_{i}=\bar{v}\left[1+\gamma \sigma\left(c\left(x_{i}, t\right)-\Phi_{i}\right)\right],
$$

where we assume a Hill type response [1] $\sigma(s)=\frac{s^{2}}{k_{b}^{2}+s^{2}}$ to represent the stress of the difference between external and adapted chemical concentrations $s=c-\Phi$. Although the form of $\sigma(s)$ would be hard to determine from the experimental data, we expect that the stress would monotonically increase as the discrepancy increases between adapted and exposed concentrations; however, there might be saturation at large stress limits as assumed in Eq. (4).

In the numerical simulations, parameters related to turning rate and swimming velocity were adjusted to the values obtained from the experiment. The diffusion coefficient of the chemoattractant was assumed to be of the same order as that of free water molecules at room temperature. We also assumed that both the decay time of the chemical and internal memory were of the order of minutes.

Without the velocity change due to the chemical stress, i.e., $\gamma=0$, above a critical initial density of cells, a stable aggregation with a cusplike peak appears, where the cell density is not uniform inside the aggregation [Fig. 8(c)]. Position of the peak is immobile during the aggregation process of cells.

Once the velocity control associated with internal memory was included, the boundaries between high and lowdensity regions had a steeper slope, and the density profile inside the aggregation became rounded. In addition to the shape of the density distribution, the dynamics of the population drastically changed; as seen for the "mobile belt" (Fig. 2 ), the boundary of the aggregate moved slowly, accompanied by nucleation and coalescence [Fig. 8(b)].

Adaptation time $\tau$ is a crucial parameter in the present model. If $\tau$ is shorter than the turn interval (typically $10 \mathrm{~s}$ in experiments), there is no qualitative change in behavior; the rounded profile of cell density and translation of the aggregation are not seen for $\tau \ll 1 / \mu$. Furthermore, we expect that $\tau \bar{v}(\bar{v}$ the average swimming velocity) would give the characteristic length scale of cell distribution which was comparable to interaggregation distance and/or aggregation size. Both estimation of parameters with statistical analysis and quantitative comparison of the present model with experiments are our future subject.

\section{CONCLUSION}

In summary, we observed distinctive swarming dynamics of clusters of paramecia including stationary, translational, and unstable modes of a high-density belt. On the basis of

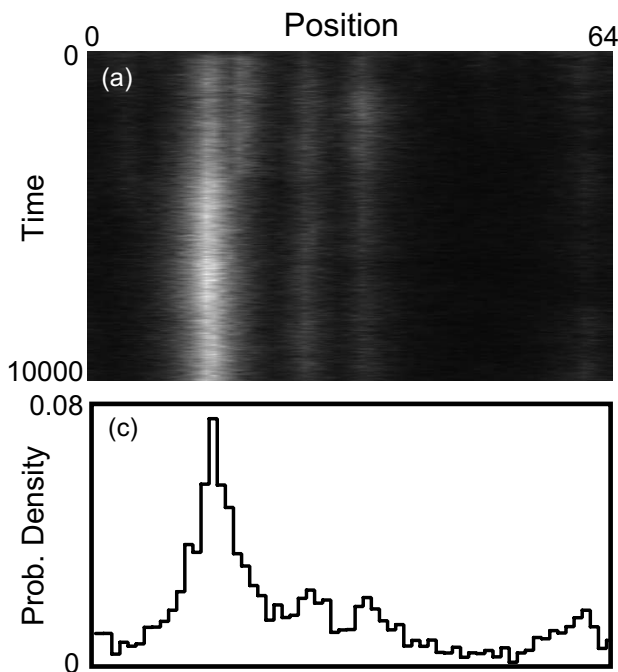

Position
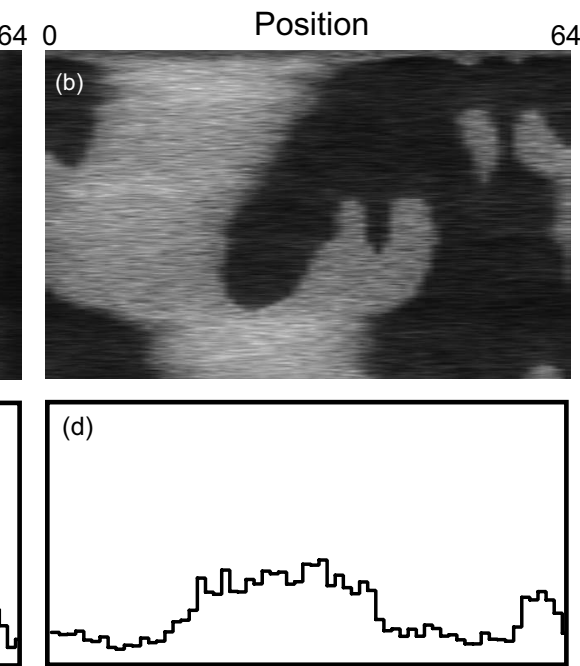

Position
FIG. 8. Evolution of cell density (upper row) and its snapshot (lower row) obtained from numerical simulations of the present model. (a) and (c) Without the velocity control effect $(\gamma=0)$ and $(b)$ and (d) $\gamma=2$. Other parameters were $\bar{v}=0.7, \bar{\mu}=0.02, \lambda=20, k_{a}$ $=5, D_{a}=0.002, \zeta=1.0, \alpha=0.02, \tau$ $=30$, and $k_{b}=20$. To make these plots, 1000 random walkers were used. The snapshots were taken after 10000 time steps. 
analysis of swimming trajectories from experimental data, we also proposed a model that includes a velocity change due to a simplified adaptation process as well as a turn rate control process, and numerical simulations of the model indicated that the slow dynamics of the swarm emerged within the framework of the present assumptions. The present results suggest possibilities of adaptation-induced diversity in swarming behavior.

In the present study, we have focused on the behavior of protozoan in a tubular geometry. Even if the size of the ring is doubled (100 $\mathrm{mm}$ in circumference), density profile along the tube as a function of initial density is qualitatively the same, thus, we expect that the effect of carvature is not significant. However, the number of clusters became larger compared to the same density in the shorter tube. So that there might be a selection mechanism of aggregation size and/or interdistance between aggregation through chemotaxis associated with adaptation. In the two-dimensional case, we also observed spontaneous aggregation of cells at high cell density, which can be seen as spots. Unfortunately, such spots are mainly initiated at/near the boundaries of container and we could not exclude the finite boundary effect on the aggregation process. However, we believe that the present study for quasi-one-dimensional geometry can be generalized to more "natural" cases including three-dimensional geometry.

In a biological context, in order to survive a harsh environment, stable cluster forming process may cause a serious disadvantage for micro-organisms, so that there has to be some "self-disassemble" mechanism in a long time scale. We suppose that the coupling of slow adaptation and quick response to environmental change is the key to understand such a self-disassembled mechanism of clusters.

\section{ACKNOWLEDGMENTS}

We thank Y. Nakaoka and H. Miyata for the set up of the experimental system, continuous discussion and encouragement. We also thank T. Kawakatsu for helpful discussions.
[1] J. D. Murray, Mathematical Biology (Springer-Verlag, Berlin, 1989).

[2] L. Tsimring, H. Levine, I. Aranson, E. Ben-Jacob, I. Cohen, O. Shochet, and W. N. Reynolds, Phys. Rev. Lett. 75, 1859 (1995).

[3] H. Salman, A. Zilman, C. Loverdo, M. Jeffroy, and A. Libchaber, Phys. Rev. Lett. 97, 118101 (2006).

[4] E. Keller and L. Segal, J. Theor. Biol. 30, 500 (1971).

[5] T. Hillen and H. G. Othmer, SIAM J. Appl. Math. 61, 61 (2000); H. G. Othmer and T. Hillen, ibid. 62, 1222 (2002).

[6] F. Oosawa and Y. Nakaoka, J. Theor. Biol. 66, 747 (1977).
[7] C. Kung and Y. Saimi, Annu. Rev. Physiol. 44, 519 (1982).

[8] H. S. Jennings, Behavior of the Lower Organisms (Indiana University Press, Bloomington, 1906).

[9] Y. Naitoh and K. Sugino, J. Protozool 31, 31 (1984).

[10] K. Tawada and F. Oosawa, J. Protozool 19, 53 (1972).

[11] Y. Nakaoka, H. Tokui, Y. Gion, S. Inoue, and F. Oosawa, Proc. Jpn. Acad., Ser. B: Phys. Biol. Sci. 58, 213 (1982).

[12] Y. Nakaoka and F. Oosawa, J. Protozool 24, 575 (1977).

[13] P. M. Simon and H. A. Gutowitz, Phys. Rev. E 57, 2441 (1998). 